\title{
Récepteur de type I de la mélanocortine, risque de cancers cutanés, et sensibilité aux ultraviolets
}

La pigmentation cutanée joue un rôle important dans la protection contre les rayonnements ultraviolets (UVA et UVB), qui sont les facteurs carcinogènes majeurs de la peau. En effet, les études cliniques montrent que l'incidence des cancers cutanés, mélanomes et carcinomes, dépend du type de peau (le risque étant maximal pour les peaux claires ne bronzant pas) et augmente avec l'ensoleillement.

La pigmentation cutanée reflète le taux de synthèse de mélanine par les mélanocytes ainsi que son transfert aux kératinocytes. On distingue deux types de mélanine dans la peau humaine : l'eumélanine, noire et photoprotectrice, et la phaeomélanine, jaune, qui pourrait au contraire être pro-carcinogène car elle produit des radicaux libres après exposition au rayonnement ultraviolet. La synthèse et la proportion relative des mélanines sont contrôlées génétiquement. L'hormone mélanotrope $\alpha$-MSH (melanocyte stimulating hormone) dont le précurseur est la pro-opiomélanocortine (POMC) est l'hormone clé impliquée dans la mélanogenèse. Son récepteur MC1R est une protéine de 317 acides aminés de la famille des récepteurs à 7 domaines couplés à la protéine $G$, et est exprimé dans plusieurs types cellulaires en particulier les mélanocytes. La fixation de l' $\alpha$-MSH sur MC1R entraîne une augmentation de la concentration d'AMP cyclique intracellulaire et de l'activité PKA. Cela a pour effet: (1) une stimulation de la mélanogenèse avec augmentation de la synthèse d'eumélanine aux dépens de la phaeomélanine; (2) un effet mitogène sur les mélanocytes [1]. se fixe également avec une haute affinité sur ce récepteur avec les mêmes effets que l' $\alpha$-MSH.

Le gène $M C 1 R$ est localisé en $16 q 24.3$ et est constitué d'un seul exon. Une vingtaine de polymorphismes ont été récemment identifiés, dont la majorité sont localisés dans une région de 42 acides aminés dans le second domaine transmembranaire. Chez la souris, les variants récessifs entrâ̂nent une perte de fonction du récepteur et sont associés à une production excessive de phaeomélanine et donc des poils jaunes. Au contraire, les variants dominants entraînent "un gain de fonction ", avec augmentation de la production de pigment noir et diminution des taux de pigments jaunes [2, 3]. Chez l'homme, des variants entraînant une perte de fonction ont été aussi identifiés (Val60Leu, Arg142His, Arg151Cys, Arg160Trp et Asp294 His). Ces variants ne peuvent stimuler la production d'AMP cyclique aussi efficacement que le récepteur sauvage [4, $5]$; ils entraînent une diminution d'affinité pour l' $\alpha$-MSH et une synthèse accrue de phaeomélanine aux dépens de l'eumélanine [6].

Plusieurs études antérieures avaient montré que certains allèles de $M C 1 R$ (Arg151Cys, Arg160Trp et Asp294 His) étaient très fréquemment retrouvés chez les individus ayant les cheveux roux et un phototype clair (I et II) [7], qui ont aussi un risque plus élevé de mélanome. De plus, certains variants de $M C 1 R$ sont aussi associés à une faible capacité de bronzage, et ce, indépendamment de la couleur des cheveux [8].

D'autres travaux ont ensuite montré que des variants de MC1R pourraient être associés au risque de développe- ment de mélanome. En 1996, une première étude révélait une association entre le polymorphisme (Asp84Glu) du gène MC1R et le risque de mélanome [9]. Dans une grande étude cas-témoins australienne récente, on observait des variants chez $72 \%$ des individus ayant un mélanome, contre seulement $56 \%$ des sujets témoins, la différence étant surtout significative pour les malades de phototype III ou IV [10]. En outre, le risque de mélanome doublait chaque fois qu'un allèle variant supplémentaire était présent.

Jusqu'à très récemment, le rôle des variants du gène MCIR comme facteur de risque des cancers cutanés autres que les mélanomes, c'est-àdire les carcinomes, n'était que mal connu. Les carcinomes cutanés comprennent les carcinomes basocelullaires (BCC) et spinocellulaires (SCC), et sont les cancers les plus fréquents chez l'adulte $(30 \%$ de tous les cancers). Ils représentent par leur multiplicité et leur tendance à récidiver un grave problème de santé publique. Une meilleure connaissance des facteurs de prédisposition à ces cancers cutanés permettrait de mieux définir les stratégies de prévention de ces tumeurs.

Si un polymorphisme Asp294His de MCIR avait bien été retrouvé de façon plus fréquente chez les patients atteints de carcinome, la petite série de cette étude conduite au RoyaumeUni limitait l'impact de ce résultat [11]. Deux publications récentes [12, 13] permettent maintenant d'évaluer de façon plus large l'association des variants du gène $M C 1 R$ avec le risque de développement des carcinomes cutanés. 
La première, qui a porté sur un grand nombre de sujets (838 dont 453 patients atteints de carcinome et 385 témoins) a révélé l'existence d'au moins 27 variants du gène $M C 1 R$ [12]. Les auteurs retrouvent une association nette entre les caractères peau claire et cheveux roux et les variants Arg151Cys et Arg160Trp, plus faible avec le variant Asp294His. D'une façon globale, et indépendamment du type de variant, les porteurs de deux variants étaient prédisposés au développement de carcinome cutané comparativement aux porteurs de deux allèles sauvages, le risque des individus porteurs d'un seul variant étant intermédiaire. Le risque individuel de chaque variant a pu être évalué et huit variants à risque ont été retrouvés: Asp84Glu, His260Pro, Asp294His, et à un moindre degré Val60leu, Val92Met, Arg142His, Arg151Cys et Arg160Trp. Enfin, le risque associé aux variants du gène $M C R 1$ était indépendant du type de peau (excepté pour les variants Arg151Cys et Arg160Trp).

La seconde étude, réalisée sur un groupe plus restreint de patients (220 dont 111 porteurs d'au moins un carcinome ou une lésion précancéreuse, et 109 témoins), n'a pas permis d'étudier le risque individuel de chaque variant [13]. Cependant, en rassemblant tous les allèles variants, les auteurs montraient, comme dans l'étude précédente, une association importante avec les carcinomes cutanés, le risque étant intermédiaire chez les sujets porteurs d'un seul allèle variant. Ici encore, le risque de cancer persistait après ajustement pour le type de peau.
Ces résultats montrent clairement que certains variants du gène $M C 1 R$ sont un facteur important de prédisposition au développement de cancers cutanés. Cependant, ce risque n'est apparemment pas dû simplement au phénotype cutané, pigmenté ou non. D’autres mécanismes que ceux qui sont impliqués dans la mélanogenèse doivent donc intervenir dans cette relation entre les polymorphismes de $M C 1 R$ et le risque de développement des cancers cutanés. Les fonctions anti-inflammatoires et immunomodulatrices de l' $\alpha$-MSH ainsi que ses fonctions de contrôle de la croissance des mélanocytes et des kératinocytes pourraient être des pistes intéressantes à explorer pour expliquer les conséquences biologiques de ces variants en cancérogenèse cutanée.

1. Bertolotto C, Busca R, Ballotti R, Ortonne JP. L'AMP cyclique est un régulateur de la pigmentation de la peau. Med Sci $2000 ; 17: 177-85$. 2. Cone R, Lu D, Koppula S, et al. The melanocortin receptors: agonist, antagonist and the hormonal control of pigmentation. Rec Prog Horm Res $1996 ; 51: 287-318$.

3. Robbins LS, Nadeau JH, Johnson KR, et al. Pigmentation phenotyes of variant extension locus alleles result from point mutations that alter MSH receptor function. Cell 1993; 72: 827-34. 4. Frandberg PA, Doufexis M, Kapas S, Chhajlani V. Human pigmentation phenotype: a point mutation generates nonfunctional MSH receptor. Biochem Biophys Res Commun 1998; 245: 490-2.

5. Schioth HB, Phillips SR, Rudzish R, BirchMachin MA, Wikberg JE, Rees JL. Loss of function mutations of the human melanocortin 1 receptor are common and associated with red hair. Biochem Biophys Res Commun 1999; 260 : 488-91.

6. Xu X, Thornwall M, Lundin LG, Chhajlani V. Val92Met variant of the melanocyte stimulating hormone receptor gene. Nat Genet 1996; 14 : 384. 7. Valverde P, Healy E, Jackson I, Rees JL, Thody AJ. variants of the melanocyte-stimulating hormone receptor gene are associated with red hair and fair skin in humans. Nat Genet 1995; 11 : 32830 .

8. Healy E, Flannagan N, Ray A, et al. Melanocortin-1 receptor gene and sun sensitivity in individuals without red hair. Lancet 2000; 355 : 1072-3.

9. Valverde P, Healy E, Sikkink S, et al. The Asp84Glu variant of the melanocortin 1 receptor (MC1R) is associated with melanoma. Hum Mol Genet 1996; 5 : 1663-6.

10. Palmer JS, Duffy DL, Box NF, et al. Melanocortin-1 receptor polymorphisms and risk of melanoma: is the association explained solely by pigmentation phenotype? Am J Hum Genet 2000; 66 : 176-86.

11. Smith R, Healy E, Siddiqui S, et al. Melanocortin-1 receptor variants in an irish population. $J$ Invest Dermatol 1998; 111: 1119-22.

12. Bastiaens MT, ter Huurne JA, Kielich C, et al. Melanocortin-1 receptor gene variants determine the risk of nonmelanoma skin cancer independendly of fair skin and red hair. Am J Hum Genet 2001; 68: 884-94.

13. Box NF, Duffy DL, Irving RE, et al. Melanocortin-1 receptor genotype is a risk factor for basal and squamous cell carcinoma. J Invest Dermatol 2001 ; 116: 224-9.

\section{Nicole Basset-Séguin}

Inserm U. 532, IRP, Hôpital SaintLouis, 1, avenue Claude-Vellefaux, 75475 Paris Cedex 10, France.

\section{Nicole Soufir}

Laboratoire de Biochimie-Génétique, Hôpital Bichat-Claude-Bernard, 46, rue Henri-Huchard, 75018 Paris, France.

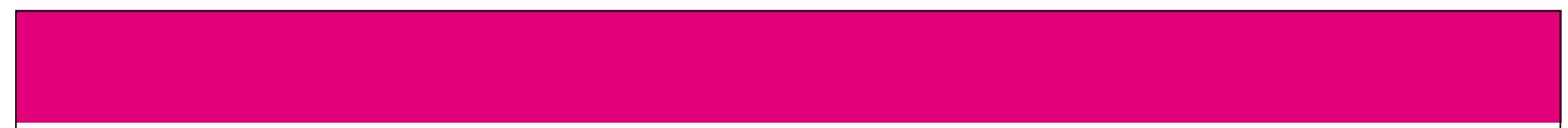

